\title{
Functional Outcome after Partial Hip Replacement for Femoral Neck Fracture (Subcapital Fracture) with Austin Moore Prosthesis
}

\section{Keren Amit ${ }^{1 *}$, Berkovich Yaron ${ }^{1}$, Lerner Alexander ${ }^{2}$, Novak Rostislav ${ }^{1}$ and Rothem David ${ }^{2}$}

${ }^{1}$ Orthopaedic Surgery A, Rambam Health Care Campus, Haifa, Israel

${ }^{2}$ Orthopaedic Surgery, Ziv medical center, Zafat, Israel Faculty of Medicine in Galilee, Bar Ilan University, Israel

\begin{abstract}
Background: Femoral neck fracture or Subcapital fracture of the hip is one of the most common fractures at old age.

There is a debate about the use of monopolar monoblock prosthesis for the treatment of those fractures. This paper is a retrospective examination of the functional and ambulation outcome of patients after partial hip replacement with cementless Austin Moore prosthesis.
\end{abstract}

Methods: Between 2002-2009, 320 patients with femoral neck fracture were treated by hip hemiarthroplasty with cementless Austin Moore prosthesis in our department. All patients had displaced femoral neck fractures. The anterolateral approach was used for all operations.

Seventy nine patients who underwent 84 hip hemiarthroplasty with cementless Austin Moore prosthesis agree to participate and were enrolled to this study. (Five patients had bilateral femoral neck fracture at separate occasions during the study period).

All patients were followed at the out patients clinic and filled a questionnaire about functional and ambulation before fracture and after rehabilitation period.

The functional outcome was divided into four levels, Poor Fair Good and Excellent functional ability. The scale of ambulation ability was 1-7 in which 1 is ambulation with no means and 7 is bed or chair ridden.

Results: The average functional score before the fracture was 22.87 and 10.43 after surgery.

The average ambulation ability before the fracture and after rehabilitation was (1.62) (4.29) respectively. Out of the 320 patients, three prosthetic hip dislocated $(0.94 \%)$, four were $(1.25 \%)$ infected and five $(1.56 \%)$ experienced a periprosthetic fracture.

Conclusion: Our study reveals that only small number of patients achieved good or excellent functional and ambulation score. The decline in functional and ambulation ability was statistical significant. We showed a relatively low incidence of complications.

This operation should be reserved only for old debilitated patients.

Keywords: Femoral neck fracture; Partial hip replacement; Austin moore prosthesis

\section{Introduction}

Femoral neck fractures of the hip are very common intracapsular fracture at old age, and occur most commonly in women older than age 65 years $[1,2]$. This fracture poses a significant health care problem, with a worldwide annual incidence of approximately 1.7 million which is expected to triple in the next 50 years as the population ages $[3,4]$. One-year mortality rates after hip subcapital fracture currently range from $14 \%$ to $36 \%$ [5]. In many cases, especially in the elderly, the medical and functional status may not return to the pre-injury levels. This imposes a great burden and expense on the medical system, and the families.

In the literature, the approach to subcapital fracture of the hip in the elderly is the subject of dispute. The adoption of either a surgical or nonsurgical approach requires a multifactorial decision that incorporates the patient's pre-injury status, including prior hip pain, ambulation, functional and mental capacity, and the complexity of their medical background [1]. Neck and Intertrochanteric fractures of the femur, as opposed to femoral shaft fractures, have a very low incidence of immediate life threatening complications such as blood loss or fat emboli. Within a few weeks after the fracture, the pain is usually tolerable and the patient can be ambulated with aid. On the other hand, in the elderly, nonsurgical treatment almost invariably leaves the patient bed or chair ridden.

Due to hip joint anatomy and physiology, the incidence of direct complications such as osteonecrosis of femoral head or fracture nonunion is very high. Furthermore the incidence of immobilityrelated, long term complications such as thromboembolic events, aspiration pneumonia and pressure sores are high, sometimes leading to preventable deaths.

*Corresponding author: Keren Amit, Orthopaedic Surgery A, Rambam Health Care Campus, P.O.B 9602, Haifa 31096, Israel, Tel: 04-8542774; Fax: 04-8542386; E-mail: amitkrn@017.net.il

Received January 24, 2014; Accepted February 22, 2014; Published February 24, 2014

Citation: Amit K, Yaron B, Alexander L, Rostislav N, David R (2014) Functional Outcome after Partial Hip Replacement for Femoral Neck Fracture (Subcapital Fracture) with Austin Moore Prosthesis. J Trauma Treat 3: 188. doi:10.4172/2167 1222.1000188

Copyright: (c) 2014 Amit K, et al. This is an open-access article distributed under the terms of the Creative Commons Attribution License, which permits unrestricted use, distribution, and reproduction in any medium, provided the original author and source are credited. 
Citation: Amit K, Yaron B, Alexander L, Rostislav N, David R (2014) Functional Outcome after Partial Hip Replacement for Femoral Neck Fracture (Subcapital Fracture) with Austin Moore Prosthesis. J Trauma Treat 3: 188. doi:10.4172/2167-1222.1000188

In the orthopedic literature, the surgical treatment of choice for displaced femoral neck fracture in elderly debilitated patients is hemiarthroplasty. The treatment of choice for good functional ability is controversial. A number of studies have examined alternative treatments such as open versus closed reduction and internal fixation. None of the implants had significantly better outcomes regarding fracture healing, osteonecrosis, wound infection, pain scores, reoperation rate, use of walking aids, periprosthetic fracture, or mortality [6,7]. Total hip arthroplasty and bipolar hemiarthroplasty are two other treatment options. Bipolar prosthesis offers no advantage over unipolar, but, the cost is much higher [8]. In our department, for elderly patients with displaced femoral neck (intracapsular) fracture we use hemiarthroplasty with cementless monopolar Austin Moore prostheses (Figure 1). For younger and active patient we prefer different device out of the options mentioned above. The criteria for each are out of the scope of this study.

We favor the anterolateral surgical approach due to low rates of dislocation and neurological injury, although there are no data supporting one approach over another [9-11]. The Austin Moore prosthesis is monopolar monoblock prosthesis. There is recent debate in the literature about the use of this prosthesis with little known about the long term results.

The aim of this study was to examine the functional and ambulation status of patients after partial hip replacement with cementless Austin Moore prosthesis.

Due to lack of information in the literature regarding different treatments and their pre and post injury functional and ambulation outcomes after this procedure, we conducted this retrospective study.

\section{Methods}

During January 2002 to April 2009, 320 patients with femoral neck fracture were treated by hip hemiarthroplasties with cementless Austin Moore prosthesis in our orthopedic department. 84 (26.25\%) men, 236 (73.75\%) women. According to our records, 170 patients have died since the operation and 150 are still alive.

During the follow up 79 patients agreed to participate in the study and to answer anonymously formal functional and ambulation questioner for the purpose of this study. This included 18 (22.5\%) men, 61 (77.5\%) women who had undergone 84 hip hemiarthroplasties with cementless Austin Moore prosthesis. Five patients had bilateral femoral neck fracture at separate occasions during the study period.

The duration of follow up was between 6 month and 7 years. The average age at operation, of the 320 patients was $83.7 \mathrm{y} / \mathrm{o}$ (Range 42.2100.2), and of the study group 83.6 y/o (57.4-97.8). Radiologically, all patients had a displaced femoral neck fracture, classified by Garden as Grade 3-4. We used the anterolateral approach for all operations, using cementless Austin Moore prosthesis at average head size of $46 \mathrm{~mm}$ (3856). The head size was measured intraoperatively.

All patients where treated as to the department Prophylactic protocol. Antibiotic, single dose preoperative and another two doses post operative of 1 gr. cefazoline. Thromboprophylaxis, prophylactic dose of $40 \mathrm{mg}$ once daily subcutaneously of LMWH from Post Operation Day 1 to 30 .

The medical problems of the analyzed group at admission included 26 (33\%) hypertension, 9 (11.4\%) diabetes mellitus, 9 (11.4\%) ischemic heart diseases, 4 (5\%) CVA/hemiparesis and 7 (9\%) arrhythmias. The average ASA scores (American Society of Anesthesiologists) of the study group was 2.7 [2-5] (Table 1).

For purposes of the study, post operative follow-up was conducted in our outpatient clinic for a 6-12 month and comprised history, functional and ambulation questioner, physical examination and radiographic evaluation. The questionnaires were answered during the out patient clinic visit, some of the patients were assisted by their escort.

Out of the 150 patient 79 agree and were able to participate in our study and filled out the questionnaires. Subsequently, details were obtained by telephone [12].

The survey consisted of a structured BADL \& IADL (Basic / Instrumental Activity Daily Living) form, which record data before the fracture and after rehabilitation period from the surgery. BADL, adapted from Katz et al. [9,12,13], included feeding, dressing, toileting, and bathing. IADL, adapted from Lawton and Brody $[9,12,14]$,

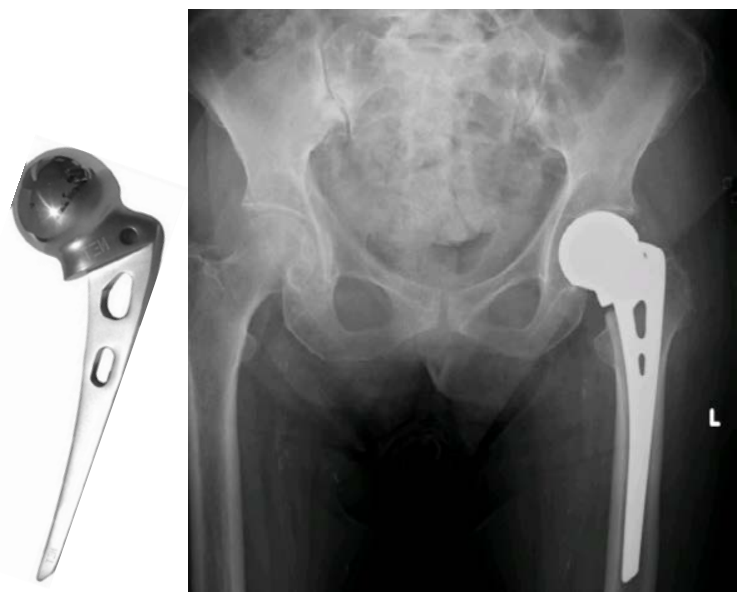

Figure 1: Hemiarthroplasty with Cementless Monopolar Austin Moore prostheses.

\begin{tabular}{|l|c|c|}
\hline Parameter & No. & $\%$ \\
\hline Age (years) & & \\
\hline mean & 83.7 & 48 \\
\hline$>85$ & & \\
\hline Gender & 61 & 77.5 \\
\hline Female & 18 & 22.5 \\
\hline Male & $3.5(6 m-7 y)$ & \\
\hline Follow up (average,years) & & \\
\hline Number of prefracture comorbidities & 38 & 48 \\
\hline 0 & 26 & 33 \\
\hline $1-2$ & 15 & 19 \\
\hline 3 and above & & \\
\hline Prefracture ambulatory status & 48 & 60 \\
\hline Independent community ambulator & 19 & 24 \\
\hline Community ambulator with cane & 10 & 13 \\
\hline Community ambulator with walker/crutches & 2 & 3 \\
\hline Household ambulator with cane 8 2.4 & 2.7 & \\
\hline American Society of Anesthesiologists & 27 & 34 \\
\hline Classification (ASA) & 50 & 63 \\
\hline I-II & 2 & 3 \\
\hline III-IV & & \\
\hline V & & \\
\hline
\end{tabular}

Table 1: Demographic and medical background data. 
Citation: Amit K, Yaron B, Alexander L, Rostislav N, David R (2014) Functional Outcome after Partial Hip Replacement for Femoral Neck Fracture (Subcapital Fracture) with Austin Moore Prosthesis. J Trauma Treat 3: 188. doi:10.4172/2167-1222.1000188

included food shopping, food preparation, performing housework, doing laundry, banking/finances, and use of public transportation.

The data were compared and analyzed, and the functional outcome was divided into four levels. A score of $0-11$ was defined as a poor functional ability; $12-24$ as fair; 25-34 as good, and 35-40 as excellent. We also examined the change in the ambulation ability on a 1-7 scale as follows: 1 . Independent community ambulatory; 2 . Community ambulator with cane; 3 . Community ambulatory with walker/crutches; 4. Independent household ambulatory; 5 . Household ambulator with cane; 6 . Household ambulator with walker/crutches; 7 . Nonfunctional ambulator $[9,13]$.

The statistical significance of the difference between the experimental groups (before fracture and after rehabilitation) was determined using an unpaired two-tailed Student $t$ test with differences considered significant at $\mathrm{p}<0.05$.

\section{Results}

The average functional score before injury was 22.87 (fair) and after surgery and rehabilitation was 10.43 (poor). The ration of preinjury functional scores to post treatment functional scores were as follows Poor - 23:54 (29\%:64\%), Fair 22:21 (28\%:25\%), Good 2:5 (2.5\%:6\%) and
Excellent 32:4 (40.5\%:5\%) respectively (Figures 2 and 3). The average preinjury ambulation ability was 1.62 , and after rehabilitation was 4.29 (Figure 4). There was statistically significant decline in functional and ambulation ability determined using Student $t$ test.

Regarding major complications, of the 320 patient records examined, 3 (0.94\%) dislocated, $4(1.25 \%)$ became infected, 5 (1.56\%) had periprostetic fracture, no thromboembolic events were record.

\section{Discussion}

We conducted a retrospective study, assessing the functional and ambulation status of elderly patients who had undergone partial hip replacement with cementless Austin Moore prosthesis. Our study reveals that only small number (9/79 or $11.5 \%)$ of patients achieved good or excellent functional score, which equates to good quality of life and the decline in ambulatory ability was significant.

In most patients the decline in functional and ambulation ability was significant.

There are, however, some methodological shortcomings to this study that might also explain these results. First, this is a retrospective study. The functional questionnaire is subjective and moreover some patients don't remember their exact functional status before the injury.

\section{Functional ability score}

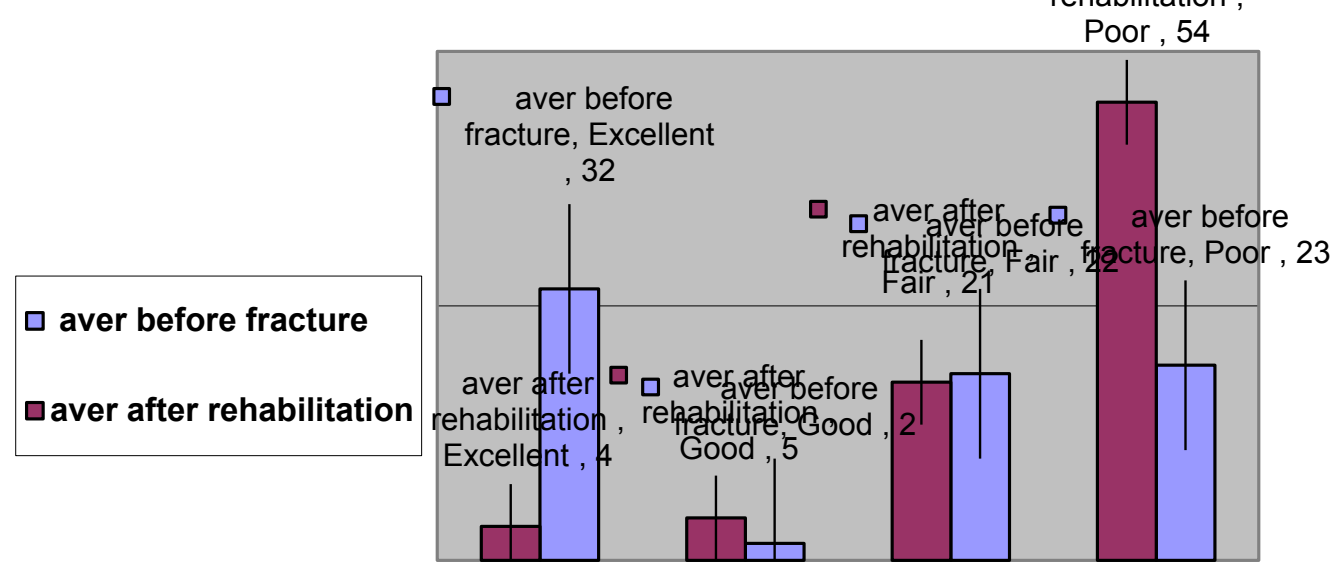

Figure 2: Functional ability score.

\section{Average functional score}

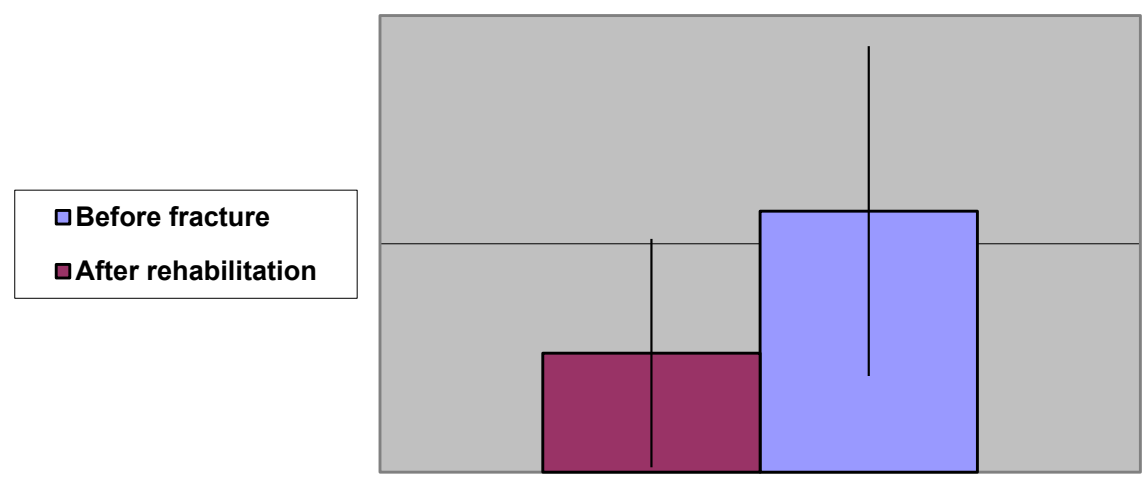

Figure 3: Average functional score. 


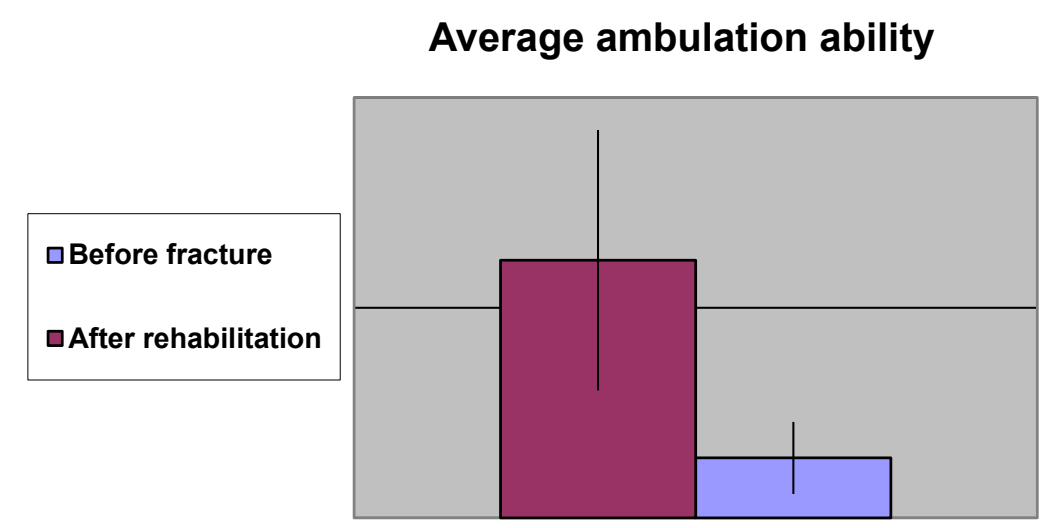

Figure 4: Average ambulation ability.

There are many factors that could influence the functional level of each patient rather than the direct results and outcome of the operation.

Most of the patients are old (mean age 83.5). Some items in the survey questionnaire, such as financial management and cooking, don't require physical abilities, but are influence by cognitive state, age and habits. It has been shown that old and cognitive dysfunction correlate with poor post-fracture ambulation [15].

Patients who were more limited in their pre-fracture ambulatory ability were more likely to regain their pre-fracture ambulation than those patients who were more independent in their pre-fracture ambulation. Multiple regression analysis has shown a significant association between pre-fracture ambulation and ambulation 1 year after fracture [16].

In this particular study group the pre injury function and ambulatory status was relatively low. Even though, we have demonstrated a significant deterioration in functional and ambulatory abilities after the hemiarthroplasty.

Those findings might be another explanation to the functional and ambulatory deterioration. The average age and long follow up are significant factors for the decline in functional and ambulation score. Multiple logistic regression analysis showed age to be a predictor of post-fracture ambulation and functional status. Koval et al. [12], in a review article, claim that age $<85$ is associated with a better outcome.

Most of our patients had significant preinjury medical problems as reflected in their ASA scores. Koval has shown that when controlled for age, the findings with respect to predictors of post-fracture ambulation are pre fracture ambulatory ability, American Society of Anesthesiologists rating of operative risk, and fracture type [12] remained the best indicators.

The fracture type is not the main subject of this work. However, when all variables were controlled, ambulatory patients in the community, who sustained an intertrochanteric fracture were less likely to become nonambulatory than those with a femoral neck fracture.

Our long term follow up demonstrates the low incidence in immobile patients of complications such as thromboembolic events, aspiration pneumonia and pressure sores. We have shown an insignificant incidence of peri-operative death and operation dependent complications such as infection, blood loss and dislocations. In addition we have demonstrated the ready ability to nurse these patients pain free, with satisfactory quality of life and is an important consideration in medical complicated patients.
This study suggests that this is a safe operation even in the elderly with significant medical problems with very low rates of immediate and late complications.

Regarding the mortality of the 170 out of the 320 patients, the one year mortality statistic after femoral neck fracture is about $30 \%$ (5) independent of the type of treatment; due to the length of time of the study this mortality rate meets the known mortality rate.

According to the Australian registry, cementless Austin Moore has a very high failure rate, compared with cemented Austin Moore. In our institute we do not cement the stems. It is possible that cementation of the Austin Moore can lead to better functional results. However cement has its own shortcomings and perils with longer operation times and increased anaphylaxis. This is subject for further investigation.

\section{Conclusion}

Hemiarthroplasty with cementless Austin Moore prosthesis is a safe operation with low incidence of perioperative and late complication. It should be reserved for elderly debilitated patients.

For younger patients or patients with good functional ability an alternative treatment should be found. THA could be a reasonable treatment and studies are now being conducted in order to evaluate this treatment.

\section{References}

1. Miyamoto RG, Kaplan KM, Levine BR, Egol KA, Zuckerman JD (2008) Surgical management of hip fractures: an evidence-based review of the literature. I: femoral neck fractures. J Am Acad Orthop Surg 16: 596-607.

2. Stevens JA, Olson S (2000) Reducing falls and resulting hip fractures among older women. MMWR Recomm Rep 49: 3-12.

3. US Census Bureau: US Interim Projections by Age, Sex, Race, and Hispanic Origin. Washington, DC: US Census Bureau, March 18, 2004. Accessed July 28, 2008.

4. Kannus P, Parkkari J, Sievänen H, Heinonen A, Vuori I, et al. (1996) Epidemiology of hip fractures. Bone 18: 57S-63S.

5. Zuckerman JD (1996) Hip fracture. N Engl J Med 334: 1519-1525.

6. Parker MJ, Stockton G (2001) Internal fixation implants for intracapsular proximal femoral fractures in adults. Cochrane Database Syst Rev: CD001467.

7. Parker MJ, Blundell C (1998) Choice of implant for internal fixation of femora neck fractures. Meta-analysis of 25 randomised trials including 4,925 patients. Acta Orthop Scand 69: 138-143.

8. Ong BC, Maurer SG, Aharonoff GB, Zuckerman JD, Koval KJ (2002) Unipolar Versus Bipolar Hemiarthroplasty: Functional Outcome After Femoral Neck 
Citation: Amit K, Yaron B, Alexander L, Rostislav N, David R (2014) Functional Outcome after Partial Hip Replacement for Femoral Neck Fracture (Subcapital Fracture) with Austin Moore Prosthesis. J Trauma Treat 3: 188. doi:10.4172/2167-1222.1000188

Page 5 of 5

Fracture at a Minimum of Thirty-six Months of Follow-up. J Orthop Trauma 16: 317-322.

9. Sikorski JM, Barrington R (1981) Internal fixation versus hemiarthroplasty for the displaced subcapital fracture of the femur: A prospective randomized study. J Bone Joint Surg Br 63: 357-361.

10. Keene GS, Parker MJ (1993) Hemiarthroplasty of the hip--the anterior or posterior approach? A comparison of surgical approaches. Injury 24: 611-613.

11. Varley J, Parker MJ (2004) Stability of hip hemiarthroplasties. Int Orthop 28: 274-277.

12. Koval KJ, Skovron ML, Aharonoff GB, Meadows SE, Zuckerman JD (1995) Ambulatory ability after hip fracture. A prospective study in geriatric patients. Clin Orthop Relat Res : 150-159.
13. Katz S, Heiple KG, Downs TD, Ford AB, Scott CP (1967) Long term course of 147 patients with fracture of the hip. Surg Gynecol Obstet 124: 1219-1230.

14. Lawton MP, Brody EM (1969) Assessment of older people: self-maintaining and instrumental activities of daily living. Gerontologist 9: 179-186.

15. Miller CW (1978) Survival and ambulation following hip fracture. J Bone Joint Surg Am 60: 930-934.

16. Mossey JM, Mutran E, Knott K, Craik R (1989) Determinants of recovery 12 months after hip fracture: the importance of psychosocial factors. Am J Public Health 79: 279-286. 